\title{
Influence of Sputtered ZnO and Al:ZnO Top Layers on Magneto-Optic Responses of Yttrium Iron Garnet Films
}

\author{
Shinichiro Mito ${ }^{1, *}$, Satsuki Kikuchi ${ }^{1}$, Yasutoshi Ito ${ }^{1}$, Nana Ota ${ }^{1}$ and Mitsuteru Inoue ${ }^{2}$ \\ 1 Department of Electronic Engineering, National Institute of Technology, Tokyo College, Hachioji, \\ Tokyo 193-1997, Japan; satsuki.kkc@gmail.com (S.K.); hibari100206@gmail.com (Y.I.); \\ 70417nana@gmail.com (N.O.) \\ 2 Department of Electrical and Electronic Information Engineering, Toyohashi University of Technology, \\ Toyohashi, Aichi 441-8580, Japan; inoue@tut.ac.jp \\ * Correspondence: mito@tokyo-ct.ac.jp; Tel.: +81-042-668-5746
}

Received: 30 August 2018; Accepted: 17 October 2018; Published: 22 October 2018

check for updates

\begin{abstract}
Zinc oxide ( $\mathrm{ZnO})$ is a promising material for combining with magneto-optic (MO) materials because it can propagate stable exciton-polaritons, with velocities considerably lower than that of photons in a vacuum. This study investigated the influence of sputtered $\mathrm{ZnO}$ and $\mathrm{Al}: \mathrm{ZnO}$ top layers on $\mathrm{MO}$ responses of a bismuth-substituted yttrium iron garnet (Bi:YIG) film. The ZnO top layer modulated the Faraday rotation and magnetic circular dichroism (MCD) of the Bi:YIG around the exciton resonance wavelength of $\mathrm{ZnO}$ at $369 \mathrm{~nm}$. Furthermore, Al-substituted $\mathrm{ZnO}$, which is a conductive $\mathrm{ZnO}$, also changed the $\mathrm{MO}$ effects around the exciton resonance wavelength. These results imply that the exciton-polaritons in $\mathrm{ZnO}$ affect the $\mathrm{MO}$ interaction, because of their considerably low group velocity. The results suggest potential for controlling the MO response via excitons.
\end{abstract}

Keywords: magneto-optical effect; magnetic garnet; zinc oxide; exciton; polariton

\section{Introduction}

The magneto-optic (MO) effect is widely used in optical applications. Recently, enhancement or modulation of the $\mathrm{MO}$ effect was reported in hybrid structures of MO materials with metallic [1-3] and dielectric materials [4-6]. These kinds of structures attract a great deal of interest because they possess advanced functional capabilities for optical devices. One method of changing $\mathrm{MO}$ responses is to localize the light in or around MO materials. Localization of light extends the time for interaction between light and MO media and, consequently, MO responses can be modulated. In earlier studies [1-6], plasmon resonance and photonic crystals were used to localize the light.

Exciton-polaritons are another promising phenomenon that localizes or slows light. An exciton is an optically excited quasiparticle formed by the electrostatic Coulomb interaction between an electron and a hole. When the semiconductor absorbs a photon, an electron can be excited into the conduction band and a hole is left behind in the valence band. This electron-hole pair is known as an exciton. Electromagnetic dipolar oscillations of excitons and photons are strongly coupled at the exciton resonance wavelength, which is called an exciton-polariton. Figure 1 shows a schematic view of an exciton-polariton. The interaction of a photon with the exciton can be expressed by Maxwell equations. The dispersion relation of the exciton-polariton is given by [7]

$$
\omega^{2}=\frac{1}{2 \varepsilon_{\infty}}\left\{c^{2} k^{2}+\varepsilon_{\infty} \omega_{L}^{2} \pm \sqrt{\left(c^{2} k^{2}+\varepsilon_{\infty} \omega_{L}^{2}\right)^{2}-4 \varepsilon_{\infty} c^{2} k^{2} \omega_{T}^{2}}\right\}
$$


where $c$ is the velocity of a photon in a vacuum, $k$ is the wavenumber, and $\omega_{T}$ and $\omega_{L}$ are longitudinal and transverse exciton frequencies. The difference between $\omega_{T}$ and $\omega_{L}$ is called longitudinal-transverse (LT) splitting, which reflects the exciton bonding energy. A dispersion relationship of the exciton-polariton approaching $\omega_{T}$ when $k \rightarrow \infty$ means the group velocity $v=\omega / k$ of exciton-polaritons is considerably slow at a wavelength of $\omega_{T}$. It potentially enlarges the interaction time and, thus, the MO responses.

The zinc oxide $(\mathrm{ZnO})$ wide-band material is an attractive material for employing exciton-polaritons. $\mathrm{ZnO}$ propagates relatively stable exciton-polaritons at room temperature because its exciton bonding energy of $60 \mathrm{meV}$ is sufficiently stronger than heat energy at a room temperature of $26 \mathrm{meV}$ [8]. One study found the experimentally measured group velocity of exciton-polaritons in $\mathrm{ZnO}$ to be $6 \times 10^{6} \mathrm{~m} / \mathrm{s}$, which is $1 / 50$ of light speed in a vacuum [9]. In a previous study, we deposited $\mathrm{ZnO}$ on bismuth-substituted yttrium iron garnet (Bi:YIG) using an electroless plating method. The electroless-plated $\mathrm{ZnO}$ slightly changed the $\mathrm{MO}$ responses [10].

In this study, $\mathrm{ZnO}$ and $\mathrm{Al}$-substituted $\mathrm{ZnO}(\mathrm{Al}: \mathrm{ZnO})$ were sputtered onto bismuth-substituted yttrium iron garnet (Bi:YIG). Magnetic circular dichroism (MCD) and Faraday rotation of the bilayers were compared with single-layered Bi:YIG.

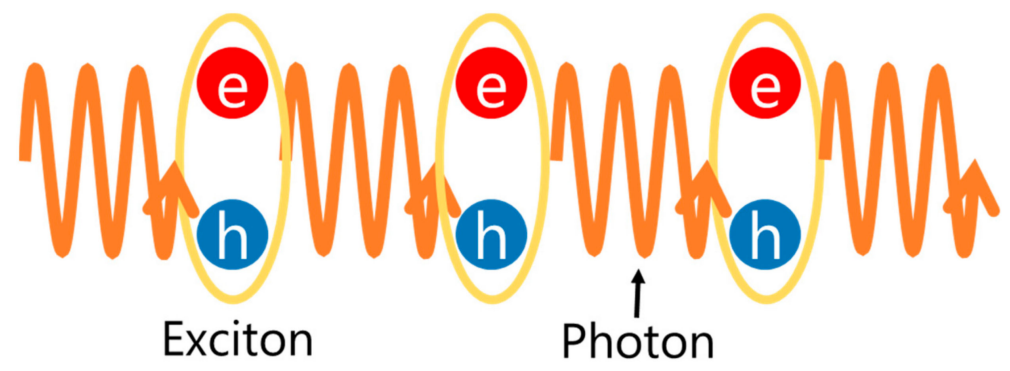

Figure 1. Schematic of an exciton-polariton.

\section{Materials and Methods}

We employed the radio-frequency magnetron sputtering method to fabricate the bilayer of $\mathrm{Bi}: Y I G$ and ZnO. Figure 2 shows a schematic of the cross-section of the fabricated bilayers. Firstly, a precursor of Bi:YIG films was deposited on an alkali-free glass substrate (Eagle XG, Corning Inc., Corning, NY, USA) by sputtering for two hours. The films were then sintered at $680{ }^{\circ} \mathrm{C}$ for $20 \mathrm{~min}$. The composition and thickness of the Bi:YIG film were $\mathrm{Bi}_{0.5} \mathrm{Y}_{2.5} \mathrm{Fe}_{5} \mathrm{O}_{12}$ and $200 \mathrm{~nm}$, respectively. Then, 200-nm-thick ZnO films were deposited onto the fabricated Bi:YIG films by sputtering. Figure 3 shows the $\mathrm{X}$-ray diffraction spectra of the fabricated $\mathrm{ZnO}$ films at various annealing temperatures. All films had good crystallinity; therefore, $\mathrm{ZnO}$ films without post-annealing were used so as to avoid a change in the characteristics of the Bi:YIG by heat-diffusion of $\mathrm{Zn}$. The single-layered $\mathrm{ZnO}$ films were formed on the glass substrate, allowing the evaluation of crystallinity and optical absorption. As with $\mathrm{ZnO}, \mathrm{Al}: \mathrm{ZnO}$ films were also fabricated and magneto-optically evaluated.

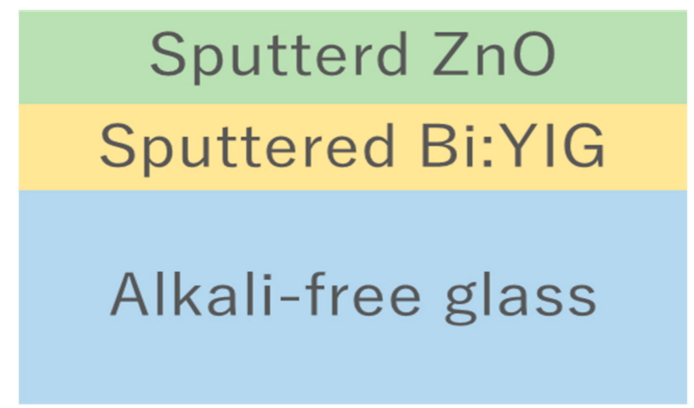

Figure 2. Representation of a cross-section of a fabricated $\mathrm{ZnO} /$ bismuth-substituted yttrium iron garnet (Bi:YIG) bilayer. 


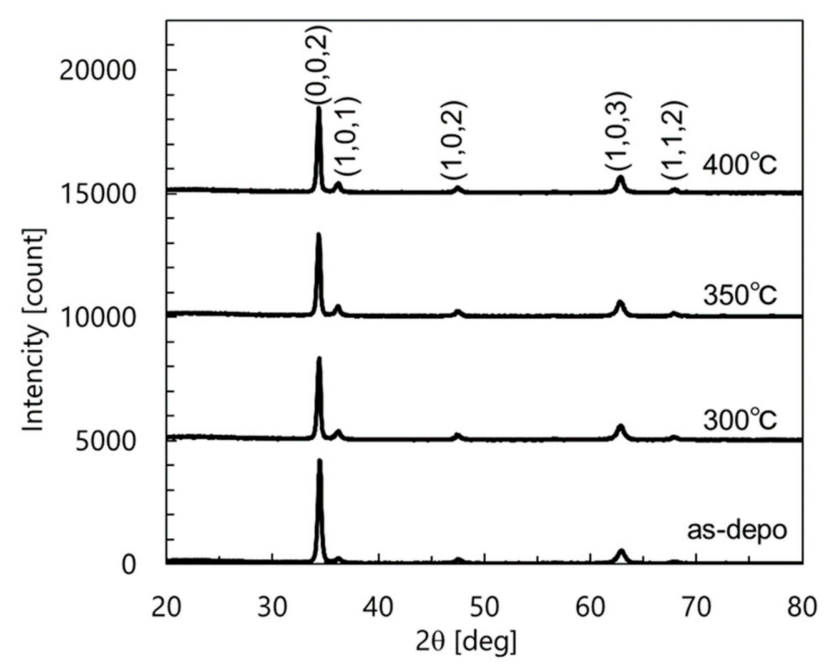

Figure 3. X-ray diffraction (XRD) spectra of $\mathrm{ZnO}$ films at various annealing temperatures.

\section{Results and Discussion}

\subsection{Bi:YIG Film with a ZnO Top Layer}

Figure 4 shows a comparison of magnetization curves of single-layered Bi:YIG and Bi:YIG with $\mathrm{ZnO}$. The circular point with a black line represents the single layer, and the cross point with a red line shows the measurement results of the fabricated bilayer. The magnetization characteristics of Bi:YIG were not changed upon formation of the $\mathrm{ZnO}$ top layer. This result means $\mathrm{Zn}$ was not significantly diffused to Bi:YIG via deposition of the ZnO. Figure 5 shows the absorption spectrum of the fabricated $\mathrm{ZnO}$. The exciton absorption peak occurred at $365 \mathrm{~nm}$, which is slightly shooter than $369 \mathrm{~nm}$ (exciton resonance wavelength of $\mathrm{ZnO}$ ). This could be because of a lattice distortion due to impurities or a lattice defect.

Figure 6 shows a comparison between the Faraday rotation spectra of Bi:YIG with ZnO and the single Bi:YIG film. The single Bi:YIG film showed a typical spectrum of magnetic garnet. In contrast. the Faraday rotation spectra of Bi:YIG with the $\mathrm{ZnO}$ top layer changed considerably around $369 \mathrm{~nm}$. This wavelength is the exciton resonance energy of $\mathrm{ZnO}$ of $3.36 \mathrm{eV}$. Small peaks occurred on both sides of $369 \mathrm{~nm}$. As the MO effect represents a phase shift of the circular polarized light, these two peaks could be due to the influence of the exciton for the left and right circular lights being different.
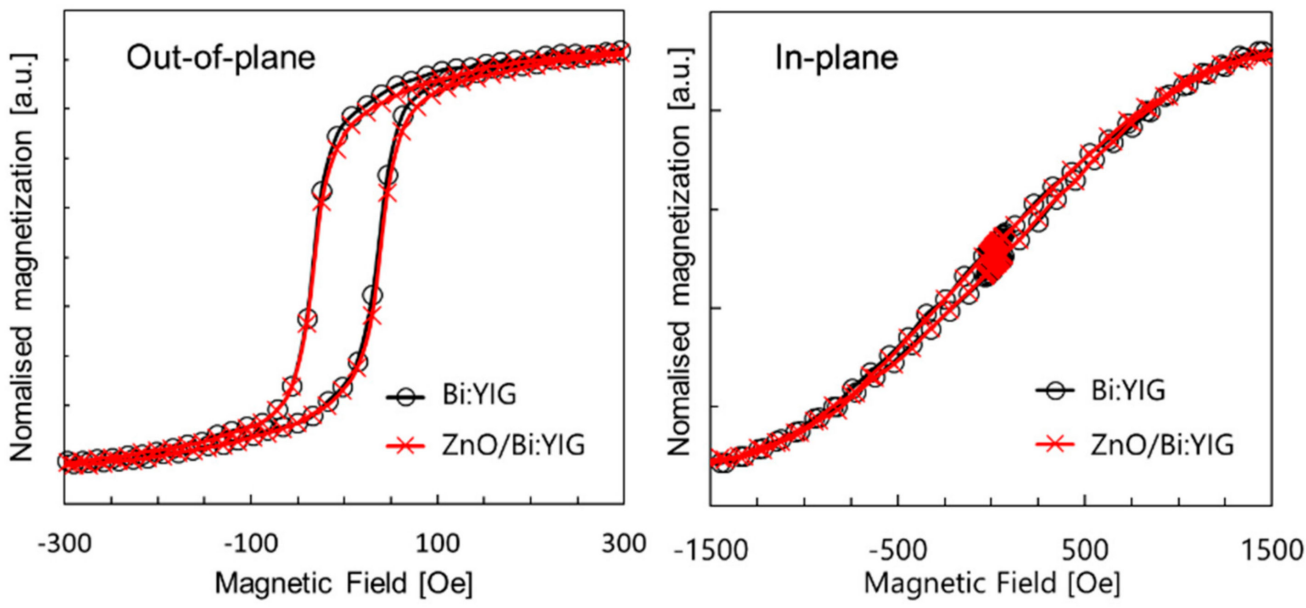

Figure 4. Comparison of the magnetization curve of single-layered Bi:YIG and Bi:YIG with ZnO. 


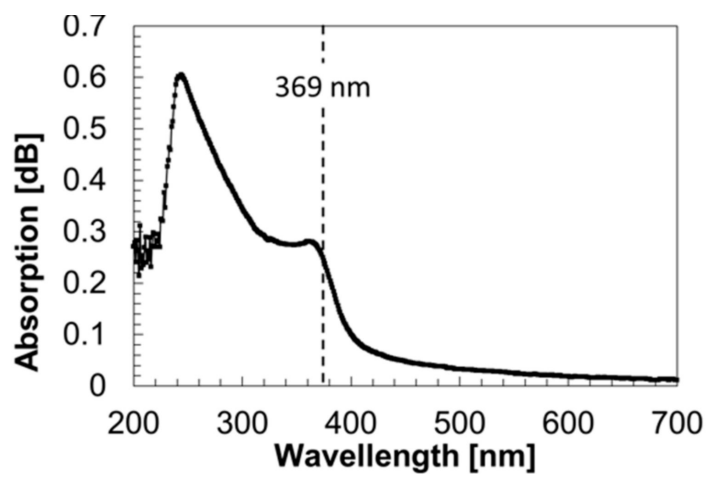

Figure 5. Absorption spectrum of the fabricated $\mathrm{ZnO}$ film.

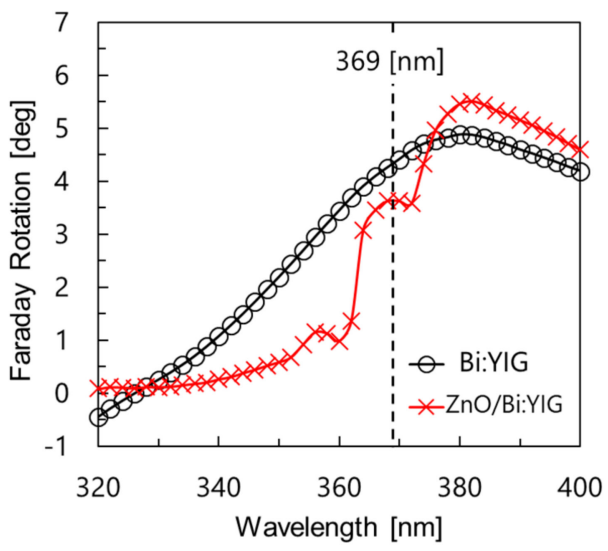

Figure 6. Faraday rotation spectra of Bi:YIG and ZnO/Bi:YIG.

Figure 7 is a comparison of the magnetic circular dichroism (MCD) spectra. As with the Faraday rotation, the MCD spectra of Bi:YIG was modulated around $369 \mathrm{~nm}$ upon depositing the $\mathrm{ZnO}$ top layer. According to Equation (1), a gradient of the dispersion relation of the exciton-polariton, which is equal to group velocity, approaches zero when the wavelength is close to exciton resonance. Therefore, these changes in MO response could be caused by the formation of the exciton-polariton and its slow group velocity. Furthermore, this change in $\mathrm{MO}$ can potentially be controlled by voltage because the exciton resonance in semiconductor systems can be altered via an electro-optic effect [11-14]. The modulation of the Faraday rotation and MCD was about 20 times bigger than that of a previous electroless-plated $\mathrm{ZnO}$ study [10]. This change could be due to the fabrication method being changed to a sputtering method, consequently increasing the density of the $\mathrm{ZnO}$ film.

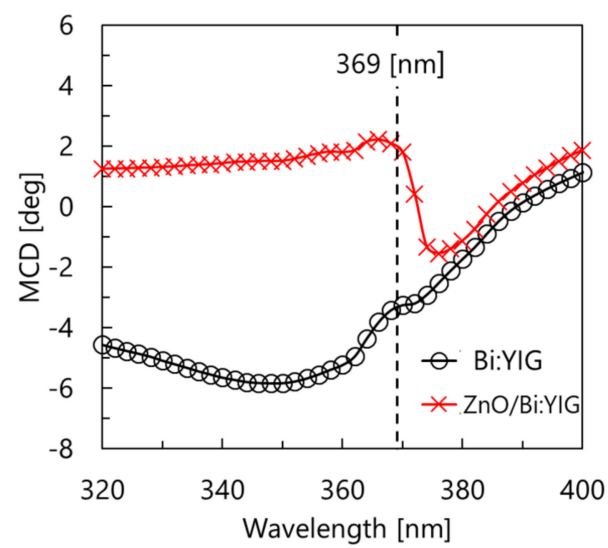

Figure 7. Magnetic circular dichroism (MCD) spectra of Bi:YIG and ZnO/Bi:YIG. 


\subsection{Bi:YIG Film with an Al-Substituted ZnO Top Layer}

$\mathrm{Al}: \mathrm{ZnO}$, which is transparent conductive $\mathrm{ZnO}$ [15], was evaluated for its ability to electrically control the exciton. $\mathrm{Al}: \mathrm{ZnO}$ was deposited and its conductivity was measured at various post-annealing temperatures. Figure 8 shows the sheet resistances of the fabricated $\mathrm{Al}: \mathrm{ZnO}$. Al: $\mathrm{ZnO}$ showed conductivity at $300{ }^{\circ} \mathrm{C}$ or lower annealing temperature. Figure 9 shows the annealing temperature dependence of the absorption spectra of $\mathrm{Al}: \mathrm{ZnO}$. Conductive $\mathrm{Al}: \mathrm{ZnO}$ does not show an absorption peak around $369 \mathrm{~nm}$; however, the insulating $\mathrm{Al}: \mathrm{ZnO}$ showed this absorption peak. The absorption peak or the optical band edge shifted to longer wavelengths as the annealing temperature increased. When the $\mathrm{ZnO}$ film is doped with $\mathrm{Al}$, the Fermi level would move into the conduction band and lead to the energy band gap widening, this effect is known as Moss-Burstein effect. The width of the optical band gap decreased as the annealing temperature increased, especially over $400{ }^{\circ} \mathrm{C}$ [16]. As a result of this effect, as-deposited $\mathrm{Al}: \mathrm{ZnO}$ showed conductivity and an optical absorption edge at short wavelengths. According to the Reference [16], this absorption edge also shifted to longer wavelengths with higher annealing temperature, similar to the present results with $\mathrm{Al}: \mathrm{ZnO}$.

Figures 10 and 11 show the Faraday rotation spectra and MCD spectra, respectively, of the $\mathrm{Al}: \mathrm{ZnO} / \mathrm{Bi}$ :YIG bilayers. Surprisingly, the bilayers showed a change in MO response around $369 \mathrm{~nm}$ at all annealing temperatures, although the measured absorption peak differed, and the sheet resistance changed from conductive to insulating. These results imply that pure $\mathrm{ZnO}$, which modulates the $\mathrm{MO}$ effects, was included in $\mathrm{Al}: \mathrm{ZnO}$. The spectrum at $700^{\circ} \mathrm{C}$ was not consistent with other spectra. The crystalline temperature of Bi:YIG is $680^{\circ} \mathrm{C}$. Therefore, crystallinity and $\mathrm{MO}$ responses of Bi:YIG changed at this temperature, resulting in the spectrum being different at $700{ }^{\circ} \mathrm{C}$.

The MO responses of Bi:YIG changed upon introducing the conductive transparent $\mathrm{Al}: \mathrm{ZnO}$. This result presents the possibility of applying an electric field to Al:ZnO/Bi:YIG and electrically controlling the $\mathrm{MO}$ response via excitons. Figure 12 shows the voltage-induced modulation of MCD of the Al:ZnO/Bi:YIG. The sample structure was a glass substrate with indium tin oxide $(100 \mathrm{~nm})$, along with Bi:YIG $(170 \mathrm{~nm})$ and the as-deposited Al:ZnO $(200 \mathrm{~nm})$. The voltage was applied from $\mathrm{Al}: \mathrm{ZnO}$ to indium tin oxide. The MCD changed slightly when $1.5 \mathrm{~V}$ was applied, and this result was reproduced three times.

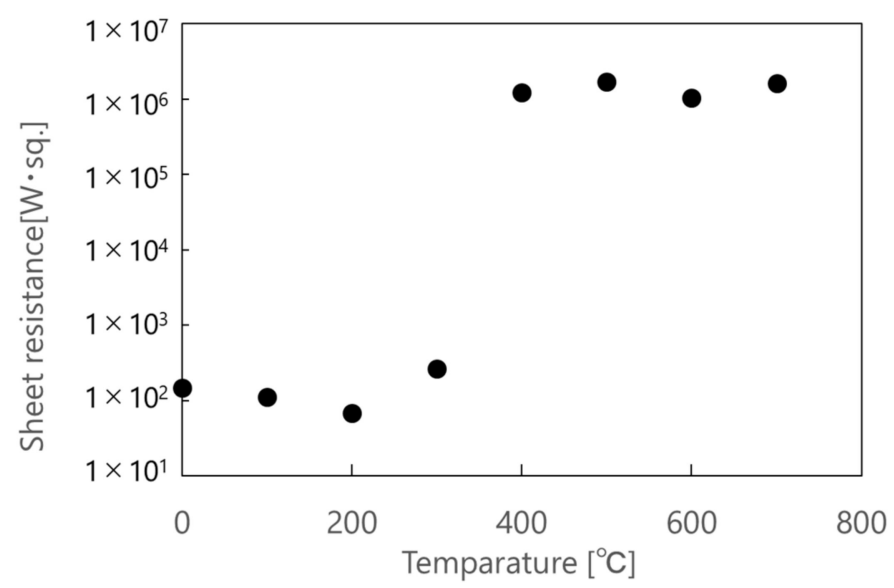

Figure 8. Sheet resistance dependence on annealing temperature of the fabricated $\mathrm{Al}: \mathrm{ZnO}$. 


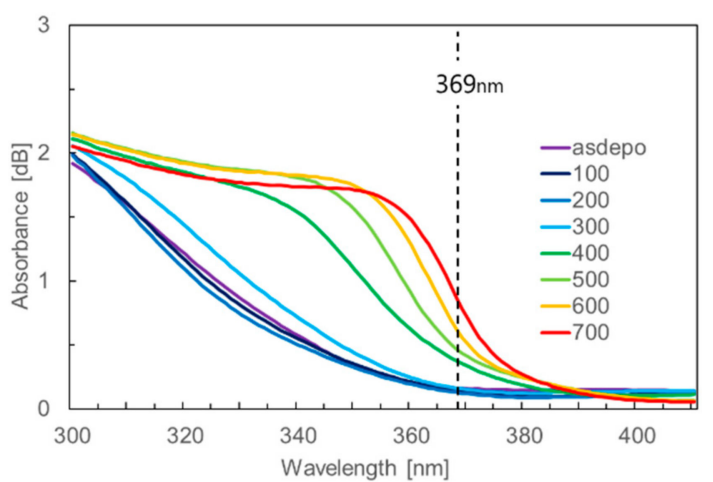

Figure 9. Absorbance spectra of the fabricated $\mathrm{Al}: \mathrm{ZnO}$ at various annealing temperatures.

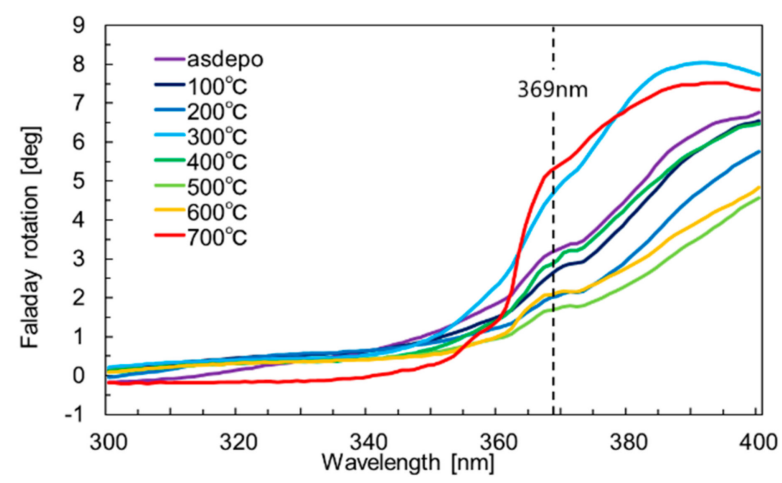

Figure 10. Faraday rotation spectra of $\mathrm{Al}: \mathrm{ZnO} / \mathrm{Bi}: \mathrm{YIG}$ bilayer at various annealing temperatures.

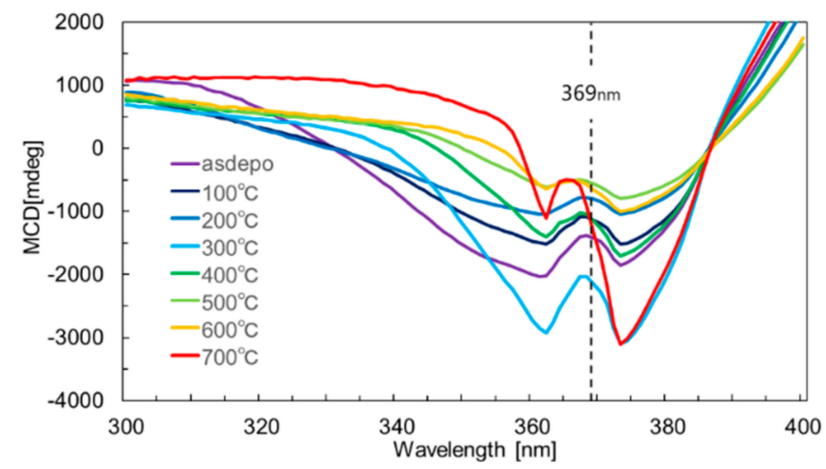

Figure 11. MCD spectra of $\mathrm{Al}: \mathrm{ZnO} / \mathrm{Bi}: \mathrm{YIG}$ bilayer at various annealing temperatures.

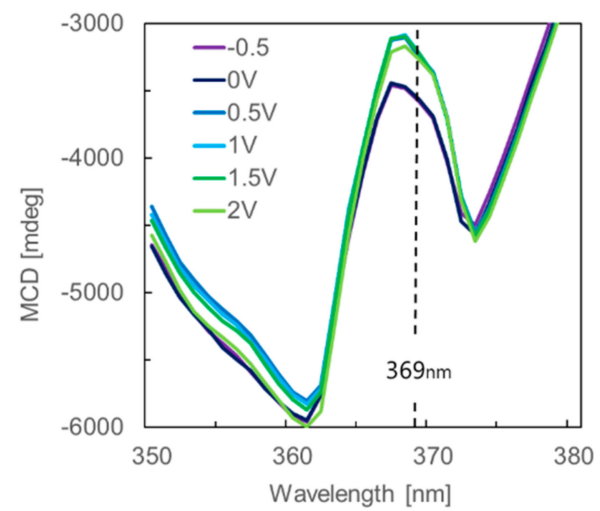

Figure 12. Voltage-induced modulation of the MCD. The sample structure was a glass substrate with indium tin oxide $(100 \mathrm{~nm})$, along with Bi:YIG $(170 \mathrm{~nm})$ and $\mathrm{Al}: \mathrm{ZnO}(200 \mathrm{~nm})$. The voltage was applied from $\mathrm{Al}: \mathrm{ZnO}$ to indium tin oxide. 


\section{Conclusions}

We investigated the influence of sputtered $\mathrm{ZnO}$ and $\mathrm{Al}: \mathrm{ZnO}$ top layers on the $\mathrm{MO}$ response of a Bi:YIG film. The ZnO top layer modulated the Faraday rotation and MCD of Bi:YIG around the exciton resonance wavelength of $\mathrm{ZnO}$ at $369 \mathrm{~nm}$. This result implies that exciton-polaritons were excited in $\mathrm{ZnO}$, and its considerably low group velocity modulated the time for interaction between photons and Bi:YIG. This change in MO effects can potentially be controlled by voltage because the exciton resonance in semiconductors can be altered via an electro-optic effect. Al-substituted $\mathrm{ZnO}$, which is conductive $\mathrm{ZnO}$, was also evaluated for its ability to electrically control the exciton. Al-substituted $\mathrm{ZnO}$ showed conductivity and modulation of $\mathrm{MO}$ response. This result offers the potential to electrically control MO response via excitons.

Author Contributions: Conceptualization, S.M.; Investigation, S.K., Y.I. and N.O.; Resources, M.I.

Funding: This work was partly supported by JSPS KAKENHI Grant Number JP16K21569 and JP26220902.

Conflicts of Interest: The authors declare no conflict of interest.

\section{References}

1. Fujikawa, R.; Baryshev, A.V.; Kim, J.; Uchida, H.; Inoue, M. Contribution of the surface plasmon resonance to optical and magneto-optical properties of a Bi:YIG-Au nanostructure. J. Appl. Phys. 2008, 103, 07D301.

2. Uchida, H.; Masuda, Y.; Fujikawa, R.; Baryshev, A.V.; Inoue, M. Large enhancement of Faraday rotation by localized surface plasmon resonance in Au nanoparticles embedded in Bi:YIG film. J. Magn. Magn. Mater. 2009, 321, 843-845. [CrossRef]

3. Uchida, K.; Adachi, H.; Kikuchi, D.; Ito, S.; Qiu, Z.; Maekawa, S.; Saitoh, E. Generation of spin currents by surface plasmon resonance. Nat. Commun. 2014, 6, 5910. [CrossRef] [PubMed]

4. Inoue, M.; Arai, K.; Fujii, T.; Abe, M. One-dimensional magnetophotonic crystals. J. Appl. Phys. 1999, 85, 5768-5770. [CrossRef]

5. Lyubchanskii, I.L.; Dadoenkova, N.N.; Lyubchanskii, M.I.; Shapovalov, E.A.; Rasing, T. Magnetic Photonic crystals. J. Phys. D Appl. Phys. 2003, 36, R277. [CrossRef]

6. Inoue, M.; Fujikawa, R.; Baryshev, A.; Khanikaev, A.; Lim, P.B.; Uchida, H.; Aktsipetrov, O.; Fedyanin, A.; Murzina, T.; Granovsky, A. Magnetophotonic crystals. J. Phys. D Appl. Phys. 2006, 39, R151. [CrossRef]

7. Ha, D.T.; Thuy, D.T.; Hoa, V.T.; Van, T.T.T.; Viet, N.T. On the theory of three types of polaritons (phonon, exciton and plasmon polaritons). J. Phys. Conf. Ser. 2017, 865, 012007. [CrossRef]

8. Ö̈zür, Ü.; Alivov, Y.I.; Liu, C.; Teke, A.; Reshchikov, M.A.; Doğan, S.; Avrutin, V.; Cho, S.-J.; Morkoç, H. A comprehensive review of $\mathrm{ZnO}$ materials and devices. J. Appl. Phys. 2005, 98, 041301. [CrossRef]

9. Xiong, G.; Wilkinson, J.; Ucer, K.B.; Williams, R.T. Time-of-flight study of bound exciton polariton dispersive propagation in ZnO. J. Phys. Condens. Matter 2005, 17, 7287. [CrossRef]

10. Mito, S.; Yusaku, S.; Sasano, J.; Takagi, H.; Inoue, M. Enhancement of magnetic circular dichroism in bi-layered ZnO-Bi:YIG thin films. AIP Adv. 2017, 7, 056316. [CrossRef]

11. Chakraborty, T. Quantum Dots-A Survey of the Properties of Artificial Atoms; Elsevier: Amsterdam, The Netherlands, 1999.

12. Miller, D.A.B; Chemla, D.S.; Damen, T.C.; Gossard, A.C.; Wiegmann, W.; Wood, T.H.; Burrus, C.A. Band-edge electroabsorption in quantum well structures: The quantum-confined stark effect. Phys. Rev. B Condens. Matter 1985, 32, 1043. [CrossRef] [PubMed]

13. Collins, R.T.; Viña, L.; Wang, W.I.; Chang, L.L.; Esaki, L.; Klitzing, K.V.; Ploog, K. Mixing between heavy-hole and light-hole excitons in GaAs/AlxGa1-xAs quantum wells in an electric field. Phys. Rev. B Condens. Matter 1987, 36, 1531. [CrossRef] [PubMed]

14. Miller, D.A.B.; Chemla, D.S.; Damen, T.C.; Gossard, A.C.; Wiegmann, W.; Wood, T.H.; Burrus, C.A. Electric field dependence of optical absorption near the band gap of quantum-well structures. Phys. Rev. Lett. 1984, 53, 2173. [CrossRef] 
15. Minami, T.; Nanto, H.; Takata, S. Highly Conductive and Transparent Aluminum Doped Zinc Oxide Thin Films Prepared by RF Magnetron Sputtering. Jpn. J. Appl. Phys. 1984, 23, L280-L282. [CrossRef]

16. Chen, Y.-Y.; Wang, P.W.; Hsu, J.-C.; Lee, C.-Y. Post-annealing properties of aluminum-doped zinc oxide films fabricated by ion beam co-sputtering. Vacuum 2013, 87, 227-231. [CrossRef] 